\title{
Exponential Synchronization of Two Nonlinearly Coupled Complex Networks with Time-Varying Delayed Dynamical Nodes
}

\author{
Wei Shao \\ School of Economics and International Trade, Zhejiang University of Finance and Economics, Hangzhou, Zhejiang 310018, China \\ Correspondence should be addressed to Wei Shao; wshao079@163.com
}

Received 25 March 2014; Revised 29 April 2014; Accepted 4 May 2014; Published 14 May 2014

Academic Editor: Xiao He

Copyright (c) 2014 Wei Shao. This is an open access article distributed under the Creative Commons Attribution License, which permits unrestricted use, distribution, and reproduction in any medium, provided the original work is properly cited.

This paper investigates the exponential synchronization between two nonlinearly coupled complex networks with time-varying delay dynamical nodes. Based on the Lyapunov stability theory, some criteria for the exponential synchronization are derived with adaptive control method. Moreover, the presented results here can also be applied to complex dynamical networks with single time delay case. Finally, numerical analysis and simulations for two nonlinearly coupled networks which are composed of the time-delayed Lorenz chaotic systems are given to demonstrate the effectiveness and feasibility of the proposed complex network synchronization scheme.

\section{Introduction}

Generally speaking, a complex network consists of a large number of interconnected nodes by edges, where a node is a fundamental unit having specific contents and exhibiting dynamical behavior, typically. As we all have known, the complex network models widely exist in real world, such as Internet, World Wide Web, biological neural networks, social connection networks, global economic markets, and ecosystems. Since the discovery of some typical complex networks such as the random networks, small-world networks and scale-free networks, many scientists and engineers from various fields, for instance, mathematics, physics, engineering, and biology, have paid increasing attention to the studies of complex networks.

In past few decades, the control and synchronization problem of networks coupled with complex dynamical systems, especially chaotic systems, has been extensively investigated in various fields due to its many potential applications [1-10]. Many kinds of synchronization have been proposed, such as complete synchronization, lag synchronization, cluster synchronization, projective synchronization, and generalized synchronization. Since chaotic systems defy synchronization, how to design effective controllers for synchronizing coupled chaotic systems becomes an important and challenging problem. Many effective methods including pinning control [11-14], adaptive control [15-20], impulsive control [21-26], and intermittent control [27-29] have been adopted to design proper controllers. Inner synchronization, that is, the synchronization of all the nodes within a network, has been investigated recently. As a matter of fact, there exist other kinds of network synchronization in real world, for example, the synchronization phenomenon between two or more complex networks, which was called outer synchronization [30]. Therefore, how to synchronize between different networks is an interesting and challenging work. Li et al. [30] pioneered in studying the outer synchronization between two unidirectionally coupled complex networks and derived analytically a criterion for them having the identical topological structures. Tang et al. [31] discussed the synchronization between two complex dynamical networks with nonidentical topological structures via using adaptive control method. Li et al. [32] studied the synchronization between two networks with different topology structures and different dynamical behaviors with open-plus-closed-loop method. Wu et al. [33] studied the problem of generalized outer synchronization 
between two complex dynamical networks with different topologies and diverse node dynamics. Li et al. [34] discussed the outer synchronization of coupled discrete-time network. The adaptive-impulsive synchronization between two complex networks with nondelayed and delayed coupling was discussed in [35], but the delay is constant; moreover, the inner connecting function is linear and the delay in the dynamical nodes is ignored. In the real world, time delays are ubiquitous in natural and artificial systems. In much of the literature, time delays in the couplings are considered; however, the time delays in the dynamical nodes, which are more complex, are still relatively unexplored. To the best of our knowledge, the problem of synchronization between two nonlinearly coupled complex networks with time-delayed dynamical nodes is seldom discussed.

Motivated by the above discussions, the aim of this paper is to discuss the problem of exponential synchronization between two nonlinearly coupled dynamical networks with identical time-delayed dynamical nodes via adaptive control. Particularly, the coupling matrices are not assumed to be symmetric or irreducible. Based on the Lyapunov function method and mathematical analysis, synchronization criteria are derived analytically. Numerical examples are used finally to illustrate the usefulness of synchronization conditions. The above proposed scheme herein will be very useful for practical engineering applications.

The rest of this paper is organized as follows. In Section 2, model description and preliminaries are given. In Section 3, some sufficient conditions for the exponential synchronization are derived with the adaptive method. In Section 4, one illustrative example is given for supporting the theoretical results. Finally, conclusions are given in Section 5.

\section{Model Description and Preliminaries}

In this paper, consider two complex dynamical networks each consisting of $N$ nonlinearly and diffusively coupled identical nodes, with each node being an $n$-dimensional dynamical system, respectively. One of the networks is characterized by

$$
\begin{aligned}
\dot{x}_{i}(t)= & f\left(t, x_{i}(t), x_{i}(t-\tau(t))\right)+\sum_{j=1}^{N} b_{i j} h\left(x_{j}(t)\right) \\
& +\sum_{j=1}^{N} c_{i j} g\left(x_{j}(t-\tau(t))\right),
\end{aligned}
$$

where, $i=1,2, \ldots, N, x_{i}(t)=\left(x_{i 1}(t), x_{i 2}(t), \ldots, x_{i n}(t)\right)^{T} \epsilon$ $R^{n}$ is the state vector of the $i$ th node, $f: R \times R^{n} \times$ $R^{n} \rightarrow R^{n}$ is a smooth nonlinear function, $\tau(t)$ is the timevarying delay. $h(\cdot) \in R^{n}$ and $g(\cdot) \in R^{n}$ are the inner connecting functions in each node. While $B=\left(b_{i j}\right)_{N \times N} \in$ $R^{N \times N}, C=\left(c_{i j}\right)_{N \times N} \in R^{N \times N}$ are the weight configuration matrices. If there is a connection from node $i$ to node $j(j \neq i)$, then the coupling $b_{i j} \neq 0, c_{i j} \neq 0$; otherwise, $b_{i j}=$ $c_{i j}=0(j \neq i)$, and the diagonal elements of matrix $B, C$ are defined as $b_{i i}=-\sum_{j=1, j \neq i}^{N} b_{i j}, \quad c_{i i}=-\sum_{j=1, j \neq i}^{N} c_{i j}$. Here, the configuration matrices are not assumed to be symmetric or irreducible.
We refer to model (1) as the drive complex dynamical network, and the response complex network with control can be rewritten in the following form:

$$
\begin{aligned}
\dot{y}_{i}(t)= & f\left(t, y_{i}(t), y_{i}(t-\tau(t))\right)+\sum_{j=1}^{N} b_{i j} h\left(y_{j}(t)\right) \\
& +\sum_{j=1}^{N} c_{i j} g\left(y_{j}(t-\tau(t))\right)+u_{i},
\end{aligned}
$$

where $y_{i}(t)=\left(y_{i 1}(t), y_{i 2}(t), \ldots, y_{i n}(t)\right)^{T} \in R^{n}$ is the response state vector of the $i$ th node and $u_{i}(i=1,2, \ldots, N)$ are the controllers to be designed later.

Before presenting the derivation of the main results, the definition, the assumptions, and lemmas are introduced as follows.

Definition 1 (see [36]). The networks (1) and (2) are said to be globally exponentially asymptotically synchronous if there exist constants $M>0$ and $\rho>0$, such that for any initial condition

$$
\left\|y_{i}(t)-x_{i}(t)\right\|=\left\|e_{i}(t)\right\| \leq M e^{-\rho t}
$$

Assumption 2. Time delay $\tau(t)$ is a differential function with $0 \leq \tau(t) \leq \tau_{M}$ and $0 \leq \dot{\tau}(t) \leq \varepsilon<1$. Clearly, this assumption includes constant delay as a special case.

Assumption 3 (see [37]). For the vector valued function $f\left(t, x_{i}(t), x_{i}(t-\tau(t))\right)$, assume that there exist positive constants $\gamma_{1}>0, \gamma_{2}>0$ such that $f$ satisfies the semi-Lipschitz condition

$$
\begin{aligned}
& (x(t)-y(t))^{T}(f(t, x(t), x(t-\tau(t))) \\
& \quad-f(t, y(t), y(t-\tau(t)))) \\
& \leq \gamma_{1}(x(t)-y(t))^{T}(x(t)-y(t)) \\
& \quad+\gamma_{2}(x(t-\tau(t))-y(t-\tau(t)))^{T}(x(t-\tau(t))-y(t-\tau(t))),
\end{aligned}
$$

for all $x, y \in R^{n}$ and $t \geq 0$.

Remark 4. Assumption 3 gives some requirements for the dynamics of isolated node in network. It is easy to verify that many chaotic systems with delays or without delays satisfy Assumption 3, for example, Chua's oscillator, Rössler's system, the Lorenz system, Chen's system, and Lü's system as well as the delayed Lorenz system, delayed Hopfield neural networks, and delayed cellular neural networks, and so on.

Lemma 5 (see [38]). For any vectors $x, y \in R^{n}$ and positive definite matrix $Q \in R^{n \times n}$, the following matrix inequality holds: $x^{T} y \leq(1 / 2)\left(x^{T} Q x+y^{T} Q^{-1} y\right)$. 


\section{Exponential Synchronization Criteria}

In this section, we will make drive-response complex dynamical networks with nondelayed and delayed coupling achieving global exponential synchronization by using adaptive controlling method.

Define the synchronization errors $e_{i}(t)=y_{i}(t)-x_{i}(t)$ $(i=1,2, \ldots, N)$; the following errors dynamics system is obtained:

$$
\begin{aligned}
\dot{e}_{i}= & f\left(t, y_{i}(t), y_{i}(t-\tau(t))\right)-f\left(t, x_{i}(t), x_{i}(t-\tau(t))\right) \\
& +\sum_{j=1}^{N} b_{i j} \bar{h}\left(e_{j}(t)\right)+\sum_{j=1}^{N} c_{i j} \bar{g}\left(e_{j}(t-\tau(t))\right)+u_{i},
\end{aligned}
$$

where $\bar{h}\left(e_{j}(t)\right)=h\left(y_{j}(t)\right)-h\left(x_{j}(t)\right), \bar{g}\left(e_{j}(t-\tau(t))\right)=g\left(y_{j}(t-\right.$ $\tau(t)))-g\left(x_{j}(t-\tau(t))\right)$.

Theorem 6. Suppose Assumptions 2 and 3 hold. The response network (2) can globally exponentially asymptotically synchronize with the driven network (1) if the controllers are designed as follows:

$$
u_{i}=\left\{\begin{aligned}
-d_{i} e_{i}(t)-\frac{N\left\|\bar{h}\left(e_{i}(t)\right)\right\|^{2}}{2\left\|e_{i}(t)\right\|^{2}} e_{i}(t) & \\
-\frac{N e^{\rho \tau_{M}}\left\|\bar{g}\left(e_{i}(t)\right)\right\|^{2}}{2(1-\varepsilon)\left\|e_{i}(t)\right\|^{2}} e_{i}(t), & \left\|e_{i}(t)\right\| \neq 0, \\
0, & \left\|e_{i}(t)\right\|=0,
\end{aligned}\right.
$$

where

$$
\dot{d}_{i}=k_{i} e^{\rho\left(t-\tau_{M}\right)}\left\|e_{i}(t)\right\|^{2}
$$

with $k_{i}$ being the known adjustable positive constants.

Proof. We construct the Lyapunov function as follows:

$$
\begin{aligned}
V(t)= & \frac{1}{2} e^{\rho\left(t-\tau_{M}\right)} \sum_{i=1}^{N} e_{i}^{T}(t) e_{i}(t) \\
& +\frac{1}{2} \sum_{i=1}^{N} \frac{1}{k_{i}}\left(d_{i}-d_{i}^{*}\right)^{2} \\
& +\frac{\gamma_{2}}{1-\varepsilon} \sum_{i=1}^{N} \int_{t-\tau(t)}^{t} e^{\rho \theta} e_{i}^{T}(\theta) e_{i}(\theta) d \theta \\
& +\frac{N}{2(1-\varepsilon)} \sum_{i=1}^{N} \int_{t-\tau(t)}^{t} e^{\rho \theta} \bar{g}^{T}\left(e_{i}(\theta)\right) \bar{g}\left(e_{i}(\theta)\right) d \theta,
\end{aligned}
$$

where $d_{i}^{*}$ is sufficiently large positive constants to be determined.
Calculate the derivative of (8) along the trajectories of (5), and with adaptive controllers (6). Thus, we obtain

$$
\begin{aligned}
\dot{V}= & \frac{1}{2} \rho e^{\rho\left(t-\tau_{M}\right)} \sum_{i=1}^{N} e_{i}^{T}(t) e_{i}(t) \\
+ & e^{\rho\left(t-\tau_{M}\right)} \sum_{i=1}^{N} e_{i}^{T}(t)\left[f\left(t, y_{i}(t), y_{i}(t-\tau(t))\right)\right. \\
& -f\left(t, x_{i}(t), x_{i}(t-\tau(t))\right) \\
& +\sum_{j=1}^{N} b_{i j} \bar{h}\left(e_{j}(t)\right) \\
& \left.+\sum_{j=1}^{N} c_{i j} \bar{g}\left(e_{j}(t-\tau(t))\right)+u_{i}\right] \\
& +\frac{\gamma_{i=1}^{N} \frac{1}{k_{i}}\left(d_{i}-d_{i}^{*}\right) \dot{d}_{i}+}{\gamma_{2}} \sum_{1-\dot{\tau}(t))}^{N} \sum_{i=1}^{\rho t} e_{i}^{T}(t) e_{i}(t) \\
& +\frac{N}{2(1-\varepsilon)} \sum_{i=1}^{\rho(t-\tau(t))} e_{i}^{T}(t-\tau(t)) e_{i}(t-\tau(t)) \\
& -\frac{N(1-\dot{\tau}(t))}{2(1-\varepsilon)} \sum_{i=1}^{N} e^{\rho(t-\tau(t))} \bar{g}^{T}\left(e_{i}(t-\tau(t))\right) \bar{g}\left(e_{i}(t-\tau(t))\right)
\end{aligned}
$$

From Assumption 2, we get

$$
\frac{1}{2} \leq \frac{1-\dot{\tau}(t)}{2(1-\varepsilon)}, \quad e^{\rho\left(\tau_{M}-\tau(t)\right)} \geq 1 .
$$

We have

$$
\begin{aligned}
\dot{V} \leq e^{\rho\left(t-\tau_{M}\right)}\left[\frac{1}{2} \rho \sum_{i=1}^{N} e_{i}^{T}(t) e_{i}(t)+\gamma_{1} \sum_{i=1}^{N} e_{i}^{T}(t) e_{i}(t)\right. \\
+\frac{\gamma_{2}}{1-\varepsilon} \sum_{i=1}^{N} e^{\rho \tau_{M}} e_{i}^{T}(t) e_{i}(t) \\
+\sum_{i=1}^{N} \sum_{j=1}^{N} b_{i j} e_{i}^{T}(t) \bar{h}\left(e_{j}(t)\right) \\
+\sum_{i=1}^{N} \sum_{j=1}^{N} c_{i j} e_{i}^{T}(t) \bar{g}\left(e_{j}(t-\tau(t))\right) \\
\quad-\sum_{i=1}^{N} d_{i}^{*}\left\|e_{i}(t)\right\|^{2}-\frac{N}{2} \sum_{i=1}^{N} \bar{h}^{T}\left(e_{i}(t)\right) \bar{h}\left(e_{i}(t)\right) \\
\\
\left.-\frac{N}{2} \sum_{i=1}^{N} \bar{g}^{T}\left(e_{i}(t-\tau(t))\right) \bar{g}\left(e_{i}(t-\tau(t))\right)\right] .
\end{aligned}
$$


From Lemma 5, we have

$$
\begin{aligned}
\sum_{i=1}^{N} \sum_{j=1}^{N} b_{i j} e_{i}^{T}(t) \bar{h}\left(e_{j}(t)\right) \leq & \frac{N^{2}}{2} \max _{1 \leq i \leq N}\left(b_{i i}^{2}\right) \sum_{i=1}^{N} e_{i}^{T}(t) e_{i}(t) \\
& +\frac{N}{2} \sum_{i=1}^{N}\left\|\bar{h}\left(e_{i}(t)\right)\right\|^{2}, \\
\sum_{i=1}^{N} \sum_{j=1}^{N} c_{i j} e_{i}^{T}(t) \bar{g}\left(e_{j}(t-\tau(t))\right) \leq & \frac{N^{2}}{2} \max _{1 \leq i \leq N}\left(c_{i i}^{2}\right) \sum_{i=1}^{N} e_{i}^{T}(t) e_{i}(t) \\
& +\frac{N}{2} \sum_{i=1}^{N}\left\|\bar{g}\left(e_{i}(t-\tau(t))\right)\right\|^{2} .
\end{aligned}
$$

Thus, we obtain

$$
\begin{array}{r}
\dot{V} \leq e^{\rho\left(t-\tau_{M}\right)} \sum_{i=1}^{N}\left[\frac{1}{2} \rho+\gamma_{1}+\frac{\gamma_{2} e^{\rho \tau_{M}}}{1-\varepsilon}+\frac{N^{2}}{2} \max _{1 \leq i \leq N}\left(a_{i i}^{2}\right)\right. \\
\left.+\frac{N^{2}}{2} \max _{1 \leq i \leq N}\left(c_{i i}^{2}\right)-d_{i}^{*}\right] e_{i}^{T}(t) e_{i}(t) .
\end{array}
$$

It is obvious that the constants $d_{i}^{*}(i=1,2, \ldots, N)$ can be properly chosen to make $(1 / 2) \rho+\gamma_{1}+\gamma_{2} e^{\rho \tau_{M}} /(1-\varepsilon)+$ $\left(N^{2} / 2\right) \max _{1 \leq i \leq N}\left(a_{i i}^{2}\right)+\left(N^{2} / 2\right) \max _{1 \leq i \leq N}\left(c_{i i}^{2}\right)-d_{i}^{*}<0$, namely, $\dot{V} \leq 0$, and then $V(t) \leq V(0)$, for any $t \geq 0$.

On the other hand, we have

$$
e^{\rho\left(t-\tau_{M}\right)} \frac{1}{2} \sum_{i=1}^{N} e_{i}^{T}(t) e_{i}(t) \leq V(t) \leq V(0) .
$$

Therefore, one has

$$
\left\|e_{i}(t)\right\| \leq \sqrt{2 V(0)} e^{-(\rho / 2)\left(t-\tau_{M}\right)} .
$$

Thus, the errors vector $e(t) \rightarrow 0$, that is, the network (1) and network (2), are globally exponentially asymptotically synchronous.

In a special case, when the networks are all linearly coupled, that is, $h\left(x_{i}(t)\right)=\Gamma_{1} x_{i}(t), g\left(x_{i}(t-\tau(t))\right)=\Gamma_{2} x_{j}(t-$ $\tau(t))$, the network (1) degenerates into the following:

$$
\begin{aligned}
\dot{x}_{i}(t)= & f\left(t, x_{i}(t), x_{i}(t-\tau(t))\right)+\sum_{j=1}^{N} b_{i j} \Gamma_{1} x_{j}(t) \\
& +\sum_{j=1}^{N} c_{i j} \Gamma_{2} x_{j}(t-\tau(t))
\end{aligned}
$$

and the response complex network with control is given by

$$
\begin{aligned}
\dot{y}_{i}(t)= & f\left(t, y_{i}(t), y_{i}(t-\tau(t))\right)+\sum_{j=1}^{N} b_{i j} \Gamma_{1} y_{j}(t) \\
& +\sum_{j=1}^{N} c_{i j} \Gamma_{2} y_{j}(t-\tau(t))+u_{i} .
\end{aligned}
$$

We have Corollary 7 for the networks (16) and (17), due to the inequality

$$
\begin{aligned}
\left\|h\left(x_{i}(t)\right)\right\| & \leq\left\|\Gamma_{1}\right\|\left\|x_{i}(t)\right\|, \\
\left\|g\left(x_{i}(t-\tau(t))\right)\right\| & \leq\left\|\Gamma_{2}\right\|\left\|x_{i}(t-\tau(t))\right\| .
\end{aligned}
$$

Corollary 7. Consider the complex networks (16) and (17), if Assumptions 2 and 3 hold. Use the following adaptive controllers and updated laws:

$$
u_{i}=\left\{\begin{array}{cc}
-d_{i} e_{i}(t)-\frac{N}{2}\left\|\Gamma_{1}\right\|^{2} e_{i}(t) & \\
-\frac{N e^{\rho \tau_{M}}}{2(1-\varepsilon)}\left\|\Gamma_{2}\right\|^{2} e_{i}(t), & \left\|e_{i}(t)\right\| \neq 0, \\
0, & \left\|e_{i}(t)\right\|=0, \\
\dot{d}_{i}=k_{i} e^{\rho\left(t-\tau_{M}\right)}\left\|e_{i}(t)\right\|^{2},
\end{array}\right.
$$

where $d_{i}$ are the feedback strength and $k_{i}>0$ are arbitrary constants. Then, the drive-response networks can globally exponentially asymptotically synchronize.

If the time-varying delay in the network (1) is the constant delay, we can obtain the following results.

Theorem 8. Suppose Assumptions 2 and 3 hold. Use the following adaptive controllers and updated laws:

$$
u_{i}=\left\{\begin{array}{cl}
-d_{i} e_{i}(t)-\frac{N\left\|\bar{h}\left(e_{i}(t)\right)\right\|^{2}}{2\left\|e_{i}(t)\right\|^{2}} e_{i}(t) & \\
-\frac{N e^{\rho \tau}\left\|\bar{g}\left(e_{i}(t)\right)\right\|^{2}}{2\left\|e_{i}(t)\right\|^{2}} e_{i}(t), & \left\|e_{i}(t)\right\| \neq 0, \\
0, & \left\|e_{i}(t)\right\|=0, \\
\dot{d}_{i}=k_{i} e^{\rho(t-\tau)}\left\|e_{i}(t)\right\|^{2}, &
\end{array}\right.
$$

where $d_{i}$ are the feedback strength and $k_{i}>0$ are arbitrary constants. Then, the drive-response networks can globally exponentially asymptotically synchronize.

Proof. We construct the Lyapunov function as follows:

$$
\begin{aligned}
V(t)= & \frac{1}{2} e^{\rho(t-\tau)} \sum_{i=1}^{N} e_{i}^{T}(t) e_{i}(t)+\frac{1}{2} \sum_{i=1}^{N} \frac{1}{k_{i}}\left(d_{i}-d_{i}^{*}\right)^{2} \\
& +\frac{\gamma_{2}}{1-\varepsilon} \sum_{i=1}^{N} \int_{t-\tau(t)}^{t} e^{\rho \theta} e_{i}^{T}(\theta) e_{i}(\theta) d \theta \\
& +\frac{N}{2(1-\varepsilon)} \sum_{i=1}^{N} \int_{t-\tau(t)}^{t} e^{\rho \theta} \bar{g}^{T}\left(e_{i}(\theta)\right) \bar{g}\left(e_{i}(\theta)\right) d \theta
\end{aligned}
$$

where $d_{i}^{*}$ is a positive constants to be determined. The rest of the proof is similar to that of Theorem 6 and is omitted here.

Similar to Corollary 7, we have Corollary 9. 
Corollary 9. Suppose Assumptions 2 and 3 hold. Use the following adaptive controllers and updated laws:

$$
\begin{aligned}
& u_{i}=\left\{\begin{array}{cl}
-d_{i} e_{i}(t)-\frac{N}{2}\left\|\Gamma_{1}\right\|^{2} e_{i}(t) & \\
-\frac{N e^{\rho \tau}}{2}\left\|\Gamma_{2}\right\|^{2} e_{i}(t), & \left\|e_{i}(t)\right\| \neq 0, \\
0, & \left\|e_{i}(t)\right\|=0,
\end{array}\right. \\
& \dot{d}_{i}=k_{i} e^{\rho(t-\tau)}\left\|e_{i}(t)\right\|^{2},
\end{aligned}
$$

where $d_{i}$ are the feedback strength and $k_{i}>0$ are arbitrary constants. Then, the drive-response network can globally exponentially asymptotically synchronize.

Remark 10. It is noted that the configuration matrices $B$ and $C$ do not need to be symmetric, diffusive, or irreducible. This means that the networks can be undirected or directed networks and may also contain isolated nodes or clusters. Therefore, the network structures here are very general and the results can be applied to a great many complex dynamical networks.

Remark 11. The feedback strengths $d_{i}$ are automatically adapted to the suitable constant, which depends on the initial values. The constants $k_{i}$ can be chosen properly to adjust the synchronization speed. The larger the constants $k_{i}$ the faster the achievement of synchronization of the driveresponse nonlinearly coupled networks with time-varying delayed dynamical nodes.

Remark 12 . Some stability criteria for the exponential synchronization between drive and response nonlinearly coupled networks with time-varying delays are derived, which can also be applied to the complex network with single time delay. Thus, the results presented in this paper improve and generalize the corresponding results of recent works. Moreover, our designed synchronization controller is not only robust but also easy to implement.

\section{Numerical Simulation}

In this section, numerical simulation is given to verify and demonstrate the effectiveness of the proposed method for exponentially synchronizing two nonlinearly coupled complex networks with time-delayed dynamical nodes. Consider the time-delayed Lorenz chaotic system as node dynamics. It is described by

$$
\begin{aligned}
f & \left(t, x_{i}(t), x_{i}(t-\tau(t))\right) \\
& =\left(\begin{array}{c}
a\left(x_{i 2}-x_{i 1}\right) \\
r x_{i 1}+(c-1) x_{i 2}-x_{i 1} x_{i 3}+c x_{i 2}(t-\tau(t)) \\
-b x_{i 3}+x_{i 1} x_{i 2}
\end{array}\right),
\end{aligned}
$$

which has a chaotic attractor when $a=10, b=8 / 3, r=28$, and $c=5$. See Figure 1 .

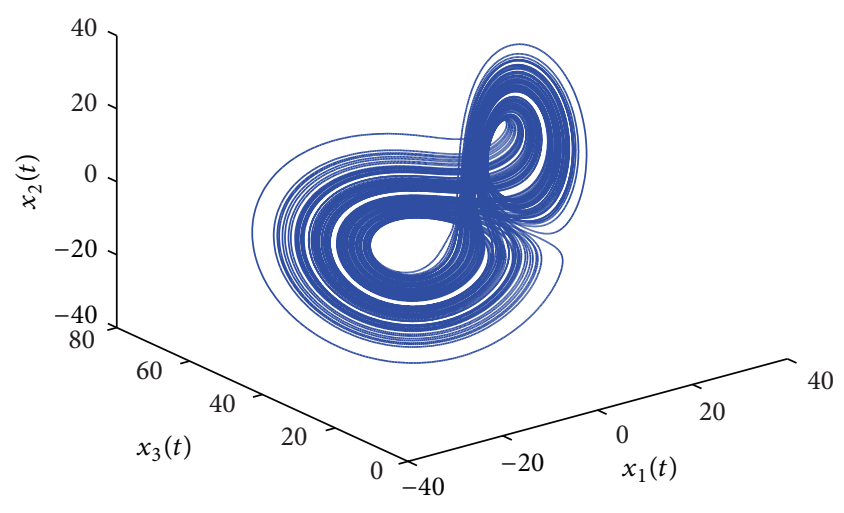

FIGURE 1: Chaos phase portrait of the time-delayed Lorenz system.

Rewrite (16) as

$f\left(t, x_{i}(t), x_{i}(t-\tau(t))\right)=A_{0} x_{i}(t)+\phi\left(t, x_{i}(t), x_{i}(t-\tau(t))\right)$,

where

$$
\begin{gathered}
A_{0}=\left(\begin{array}{ccc}
-a & a & 0 \\
r & c-1 & 0 \\
0 & 0 & -b
\end{array}\right), \\
\phi\left(t, x_{i}(t), x_{i}(t-\tau(t))\right)=\left(\begin{array}{c}
0 \\
-x_{i 1} x_{i 3}+c x_{i 2}(t-\tau(t)) \\
x_{i 1} x_{i 2}
\end{array}\right) .
\end{gathered}
$$

For any state vectors $x_{i}$ of the time-delayed Lorenz chaotic system, there exists a constant $M$ satisfying $\left\|x_{i}\right\| \leq M$ since chaotic attractor is bounded.

To satisfy Assumption 3, consider that one can always find $\eta>0$ such that $|x y| \leq \eta\left(x^{2} / 2\right)+y^{2} / 2 \eta$, and then we have

$$
\begin{aligned}
e_{i}^{T}( & t)\left[f\left(t, y_{i}(t), y_{i}(t-\tau(t))\right)-f\left(t, x_{i}(t), x_{i}(t-\tau(t))\right)\right] \\
= & e_{i}^{T} A_{0} e_{i}+e_{i 1} e_{i 3} x_{i 2}-e_{i 1} e_{i 2} x_{i 3}+5 e_{i 2} e_{i 2}(t-\tau(t)) \\
\leq & \left(-10+\eta_{1} \frac{38+M}{2}+\frac{\eta_{2} M}{2}\right) e_{i 1}^{2} \\
& +\left(4+\frac{38+M}{2 \eta_{1}}+\frac{5 \eta_{3}}{2}\right) e_{i 2}^{2} \\
& +\left(\frac{M}{2 \eta_{2}}-\frac{8}{3}\right) e_{i 3}^{2}+\frac{M}{2 \eta_{3} e_{i 2}^{2}(t-\tau(t))} \\
\leq & \gamma_{1} e_{i}^{T} e_{i}+\gamma_{2} e_{i}^{T}(t-\tau(t)) e_{i}(t-\tau(t)),
\end{aligned}
$$

where $\gamma_{1}, \gamma_{2}$ can be determined by choosing appropriate parameters $\eta_{i}>0, i=1,2,3$.

Now, we consider two nonlinearly coupled complex dynamical networks (1) and (2) with coupling delay 


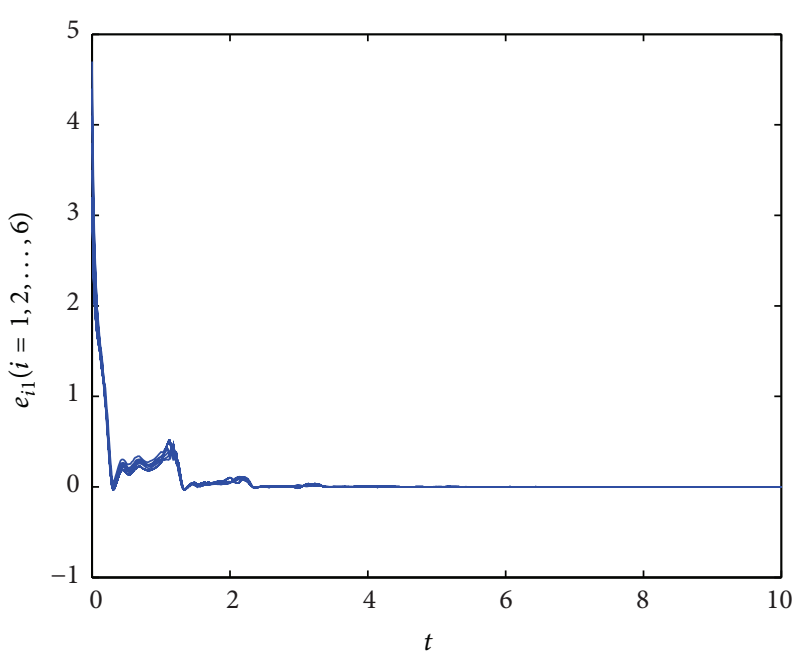

(a)

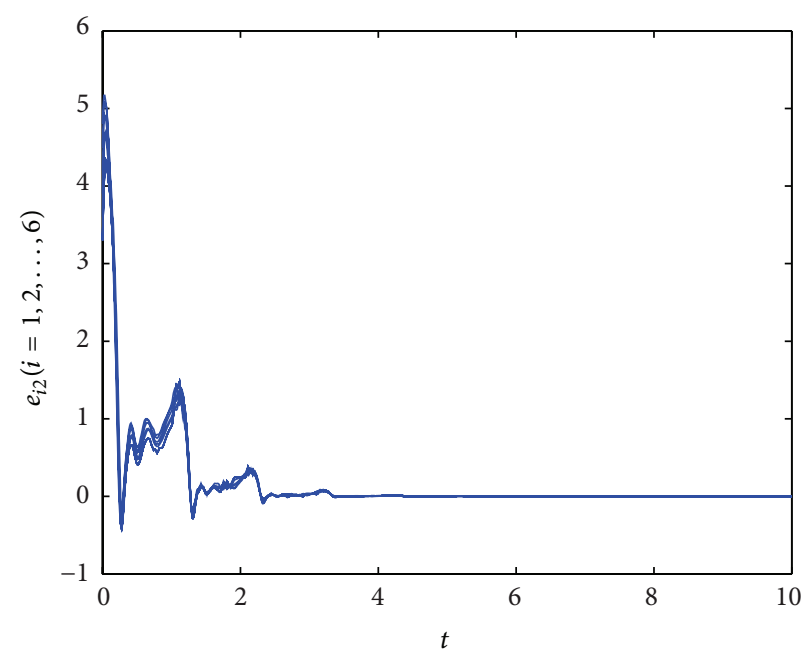

(b)

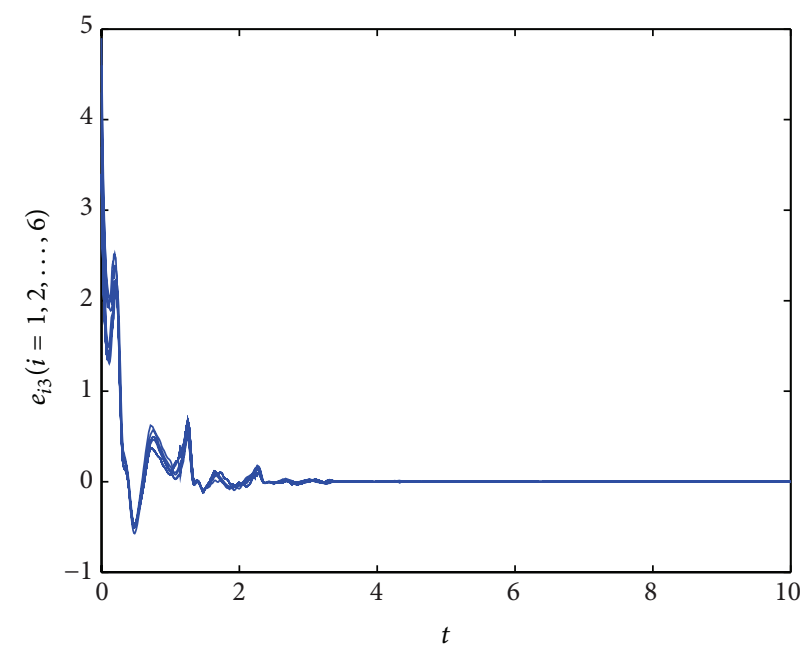

(c)

FIGURE 2: Errors of the drive-response coupled networks: (a) $e_{i 1}$; (b) $e_{i 2}$; (c) $e_{i 3}$.

consisting of 6 identical time delayed chaotic systems. Take the weight configuration coupling matrices

$$
\begin{aligned}
B & =\left(\begin{array}{cccccc}
-4 & 3 & 0 & 0 & 1 & 0 \\
1 & -6 & 2 & 0 & 0 & 3 \\
2 & 1 & -3 & 0 & 0 & 0 \\
0 & 3 & 0 & -7 & 4 & 0 \\
0 & 0 & 0 & 4 & -4 & 0 \\
1 & 0 & 1 & 0 & 0 & -2
\end{array}\right), \\
C & =\left(\begin{array}{cccccc}
-6 & 3 & 2 & 0 & 1 & 0 \\
3 & -4 & 1 & 0 & 0 & 0 \\
2 & 1 & -3 & 0 & 0 & 0 \\
1 & 0 & 0 & -2 & 0 & 1 \\
0 & 0 & 0 & 0 & -5 & 5 \\
0 & 1 & 0 & 0 & 0 & -1
\end{array}\right) .
\end{aligned}
$$

For simplicity, in the numerical simulations, we assume the time-varying delay $\tau(t)=e^{t} /\left(1+e^{t}\right)$; then $\dot{\tau}(t)=$ $e^{t} /\left(1+e^{t}\right)^{2} \in(0,1 / 2]$ satisfies Assumption 2. The initial values of the drive systems and the response systems are chosen as $x_{i}(0)=(0.1+0.1 i, 0.2+0.2 i, 0.3+0.3 i)^{T}, y_{i}(0)=$ $(-0.2+0.1 i,-0.3+0.2 i,-0.4+0.3 i)^{T}$, the positive constants $k_{i}=1$; let $h(x)=\sin (x)+3 x$ and $g(x)=-\cos (x)+$ $3 x$. Based on Theorem 6, the global exponential synchronization can be achieved. The synchronization errors are shown, respectively, in Figure 2. Figure 3 displays the state subvariables for node $i=3$ of the drive network and response networks. Figure 4 plots the total synchronization errors $\|e(t)\|=\sqrt{\sum_{i=1}^{6}\left[e_{i 1}^{2}(t)+e_{i 2}^{2}(t)+e_{i 3}^{2}(t)\right]}$ with different $k_{i}(i=1,2, \ldots, 6)$. As described in Figure 4, the larger the constants $k_{i}$ the faster the convergence to synchronization. The numerical results show that adaptive scheme for the exponential synchronization of the drive-response nonlinearly coupled complex networks is effective in all the theorems and corollaries. 


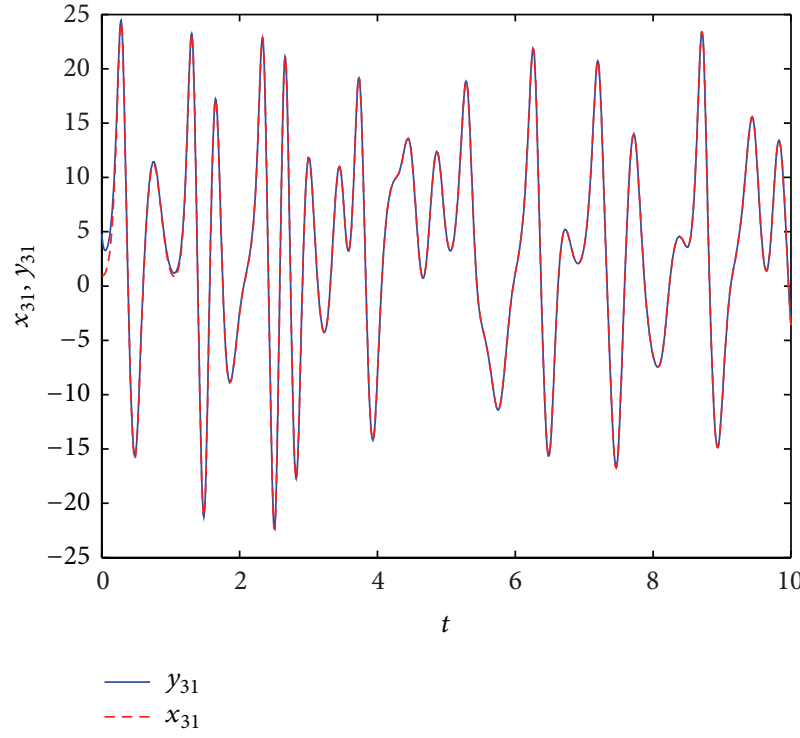

(a)

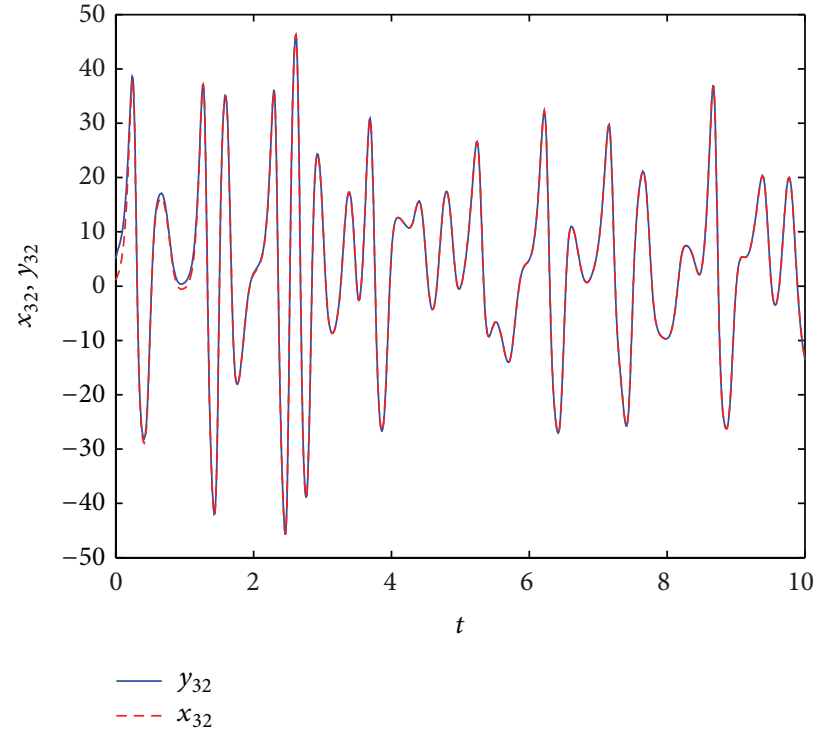

(b)

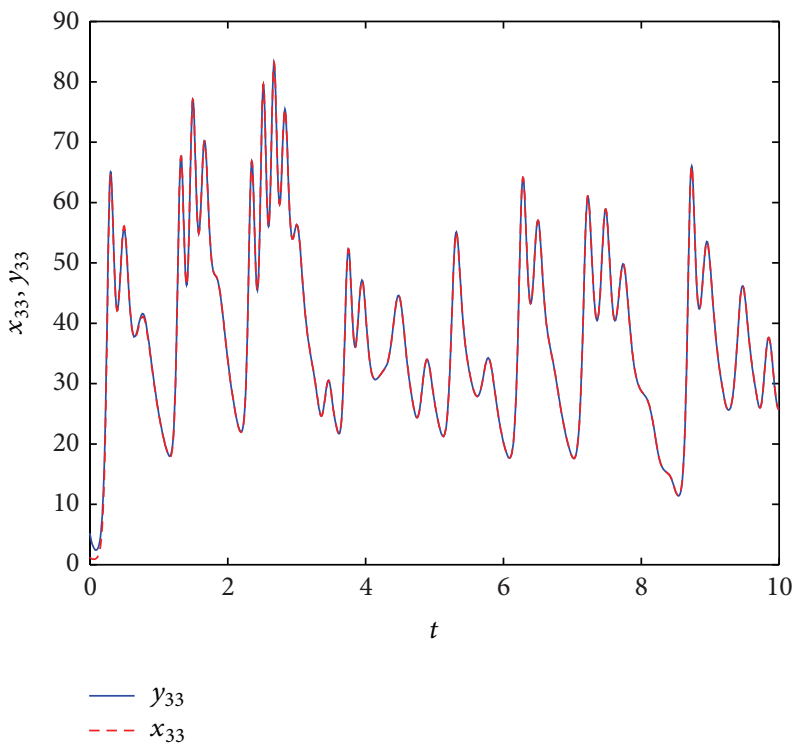

(c)

FIgURE 3: State subvariables for node $i=3$ between the drive and response coupled networks.

\section{Conclusion}

In this paper, the adaptive controllers have been proposed to study the global exponential synchronization between two nonlinearly coupled complex networks with time-varying delay dynamical nodes. By constructing the appropriate Lyapunov functions, some criteria are derived. In particular, the coupling matrices are not symmetric and irreducible. Numerical results demonstrate that the proposed approach is effective and feasible. In the analysis and simulation study of this paper, we fully considered the impact of the time delay element to the exponential synchronization of the drive-response coupled networks. In order to obtain the synchronization criteria, we did not take into account the environment factors, for example, noise, on the networks, which often affects the synchronization process of the driveresponse coupled dynamical networks. Therefore, in the near future work, we will further investigate the exponential synchronization problem of drive-response nonlinearly coupled dynamical network with noise.

\section{Conflict of Interests}

The author declares that he has no conflict of interests. 


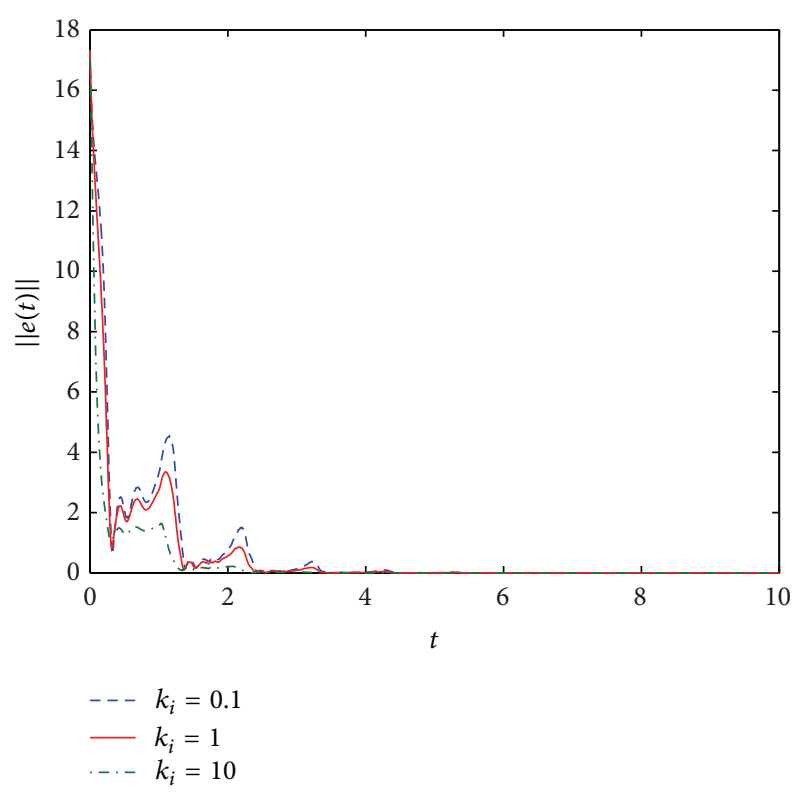

FIgURE 4: Total synchronization errors $\|e(t)\|$ with $k_{i}=0.1,1,10$.

\section{Acknowledgment}

The author would like to thank the anonymous reviewers for their invaluable comments.

\section{References}

[1] D. J. Watts and S. H. Strogatz, "Collective dynamics of 'smallworld9 networks," Nature, vol. 393, no. 6684, pp. 440-442, 1998.

[2] A. L. Barabási and R. Albert, "Emergence of scaling in random networks," Science, vol. 286, no. 5439, pp. 509-512, 1999.

[3] W. Sun, "Random walks on generalized Koch networks," Physica Scripta, vol. 88, no. 4, Article ID 045006, 2013.

[4] C. W. Wu, Synchronization in Complex Networks of Nonlinear Dynamical Systems, World Scientific, Singapore, 2007.

[5] S. Cai, J. Hao, Q. He, and Z. Liu, "Exponential synchronization of complex delayed dynamical networks via pinning periodically intermittent control," Physics Letters A: General, Atomic and Solid State Physics, vol. 375, no. 19, pp. 1965-1971, 2011.

[6] W. Sun, Y. Yang, C. Li, and Z. Liu, "Synchronization inside complex dynamical networks with double time-delays and nonlinear inner-coupling functions," International Journal of Modern Physics B, vol. 25, pp. 1531-1541, 2011.

[7] W. Sun, J. Zhang, and C. Li, "Synchronization analysis of two coupled complex networks with time delays," Discrete Dynamics in Nature and Society, vol. 2011, Article ID 209321, 12 pages, 2011.

[8] Q. Bian and H. Yao, "Generalized synchronization between two complex dynamical networks with time-varying delay and nonlinear coupling," Mathematical Problems in Engineering, vol. 2011, Article ID 978612, 15 pages, 2011.

[9] S. Zheng, "Exponential synchronization of two nonlinearly non-delayed and delayed coupled complex dynamical networks," Physica Scripta, vol. 85, no. 1, Article ID 015003, 2012.

[10] X. Wu, C. Xu, J. Feng, Y. Zhao, and X. Zhou, "Generalized projective synchronization between two different neural networks with mixed time delays," Discrete Dynamics in Nature and Society, vol. 2012, Article ID 153542, 19 pages, 2012.
[11] X. Li, X. Wang, and G. Chen, "Pinning a complex dynamical network to its equilibrium," IEEE Transactions on Circuits and Systems I: Regular Papers, vol. 51, no. 10, pp. 2074-2087, 2004.

[12] T. Chen, X. Liu, and W. Lu, "Pinning complex networks by a single controller," IEEE Transactions on Circuits and Systems I: Regular Papers, vol. 54, no. 6, pp. 1317-1326, 2007.

[13] P. DeLellis, M. Di Bernardo, and M. Porfiri, "Pinning control of complex networks via edge snapping," Chaos, vol. 21, no. 3, Article ID 033119, 2011.

[14] S. Zheng and Q. Bi, "Synchronization analysis of complex dynamical networks with delayed and non-delayed coupling based on pinning control," Physica Scripta, vol. 84, no. 2, Article ID 025008, 2011.

[15] J. Lu and J. Cao, "Adaptive synchronization of uncertain dynamical networks with delayed coupling," Nonlinear Dynamics, vol. 53, no. 1-2, pp. 107-115, 2008.

[16] S. Zheng, S. Wang, G. Dong, and Q. Bi, "Adaptive synchronization of two nonlinearly coupled complex dynamical networks with delayed coupling," Communications in Nonlinear Science and Numerical Simulation, vol. 17, no. 1, pp. 284-291, 2012.

[17] W. Yu, P. DeLellis, G. Chen, M. di Bernardo, and J. Kurths, "Distributed adaptive control of synchronization in complex networks," IEEE Transactions on Automatic Control, vol. 57, no. 8, pp. 2153-2158, 2012.

[18] M. Xiao, W. Sun, and F. Chen, "Synchronization between two discrete-time networks with mutual couplings," Abstract and Applied Analysis, vol. 2013, Article ID 167198, 6 pages, 2013.

[19] Y. Sun, W. Li, and J. Ruan, "Generalized outer synchronization between complex dynamical networks with time delay and noise perturbation," Communications in Nonlinear Science and Numerical Simulation, vol. 18, no. 4, pp. 989-998, 2013.

[20] W. Sun and S. Li, "Generalized outer synchronization between two uncertain dynamical networks," Nonlinear Dynamics, 2014.

[21] J. Zhou, L. Xiang, and Z. Liu, "Synchronization in complex delayed dynamical networks with impulsive effects," Physica A: Statistical Mechanics and Its Applications, vol. 384, no. 2, pp. 684-692, 2007.

[22] G. Zhang, Z. Liu, and Z. Ma, "Synchronization of complex dynamical networks via impulsive control," Chaos, vol. 17, no. 4, Article ID 043126, 9 pages, 2007.

[23] S. Zheng, "Projective synchronization in a driven-response dynamical network with coupling time-varying delay," Nonlinear Dynamics, vol. 69, no. 3, pp. 1429-1438, 2012.

[24] S. Zheng and W. Shao, "Mixed outer synchronization of dynamical networks with nonidentical nodes and output coupling," Nonlinear Dynamics, vol. 73, no. 4, pp. 2343-2352, 2013.

[25] S. Zheng, "Adaptive impulsive observer for outer Synchronization of delayed complex dynamical networks with output coupling," Journal of Applied Mathematics, vol. 2014, Article ID 450193, 11 pages, 2014.

[26] J. Xu and S. Zheng, "Weak projective synchronization in driveresponse dynamical networks with time-varying delay and parameter mismatch," Journal of Applied Mathematics, Article ID 356924, 8 pages, 2014.

[27] S. Cai, Z. Liu, F. Xu, and J. Shen, "Periodically intermittent controlling complex dynamical networks with time-varying delays to a desired orbit," Physics Letters. A, vol. 373, no. 42, pp. 3846-3854, 2009.

[28] S. Cai, Q. He, J. Hao, and Z. Liu, "Exponential synchronization of complex networks with nonidentical time-delayed dynamical nodes," Physics Letters. A, vol. 374, no. 25, pp. 2539-2550, 2010. 
[29] J. Mei, M. Jiang, X. Wang, J. Han, and S. Wang, "Finitetime synchronization of drive-response systems via periodically intermittent adaptive control," Journal of the Franklin Institute: Engineering and Applied Mathematics, vol. 351, no. 5, pp. 26912710, 2014.

[30] C. P. Li, W. G. Sun, and J. Kurths, "Synchronization between two coupled complex networks," Physical Review E, vol. 76, Article ID 046204, 2007.

[31] H. Tang, L. Chen, J. A. Lu, and C. K. Tse, "Adaptive synchronization between two complex networks with nonidentical topological structures," Physica A: Statistical Mechanics and Its Applications, vol. 387, no. 22, pp. 5623-5630, 2008.

[32] Y. Li, Z. R. Liu, and J. B. Zhang, "Synchronization between different networks," Chinese Physics Letters, vol. 25, no. 3, pp. 874-877, 2008.

[33] X. Wu, W. X. Zheng, and J. Zhou, "Generalized outer synchronization between complex dynamical networks," Chaos, vol. 19, no. 1, Article ID 013109, 9 pages, 2009.

[34] C. Li, C. Xu, W. Sun, J. Xu, and J. Kurths, "Outer synchronization of coupled discrete-time networks," Chaos, vol. 19, no. 1, Article ID 013106, 7 pages, 2009.

[35] S. Zheng, G. Dong, and Q. Bi, "Impulsive synchronization of complex networks with non-delayed and delayed coupling," Physics Letters A: General, Atomic and Solid State Physics, vol. 373, no. 46, pp. 4255-4259, 2009.

[36] Y. Yang and J. Cao, "Exponential synchronization of the complex dynamical networks with a coupling delay and impulsive effects," Nonlinear Analysis: Real World Applications, vol. 11, no. 3, pp. 1650-1659, 2010.

[37] L. Huang, Z. Wang, Y. Wang, and Y. Zuo, "Synchronization analysis of delayed complex networks via adaptive time-varying coupling strengths," Physics Letters A, vol. 373, no. 43, pp. 39523958, 2009.

[38] J. Lu and J. Cao, "Synchronization-based approach for parameters identification in delayed chaotic neural networks," Physica A: Statistical Mechanics and Its Applications, vol. 382, no. 2, pp. 672-682, 2007. 


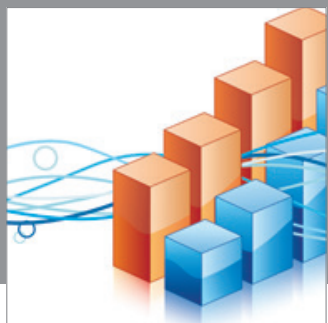

Advances in

Operations Research

mansans

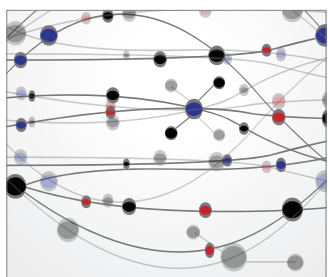

The Scientific World Journal
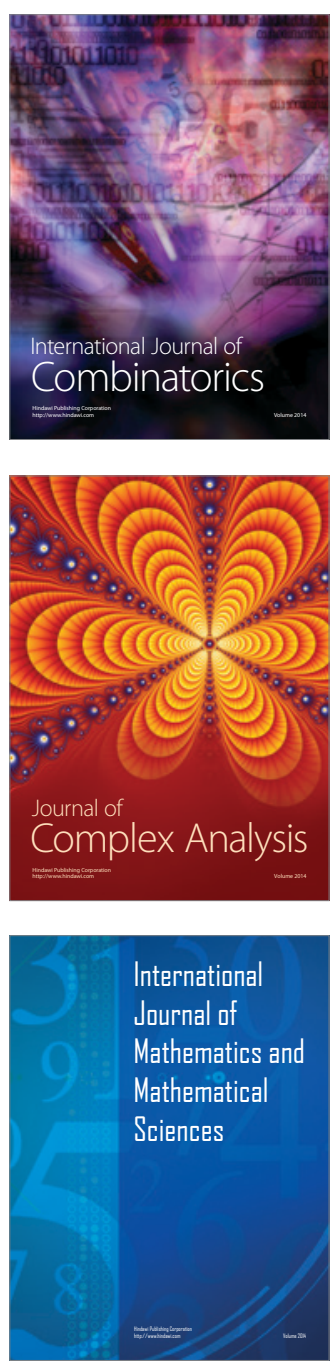
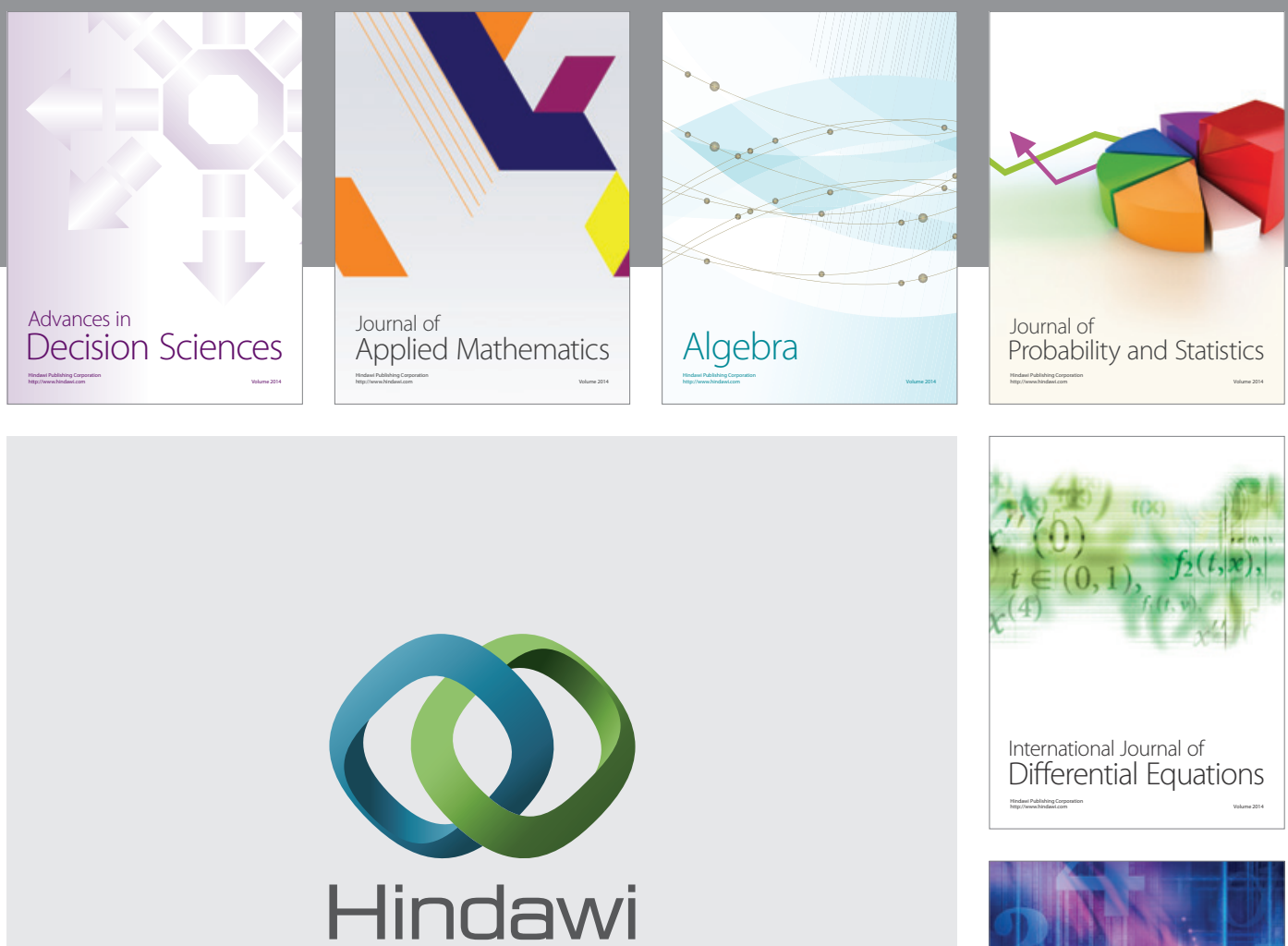

Submit your manuscripts at http://www.hindawi.com
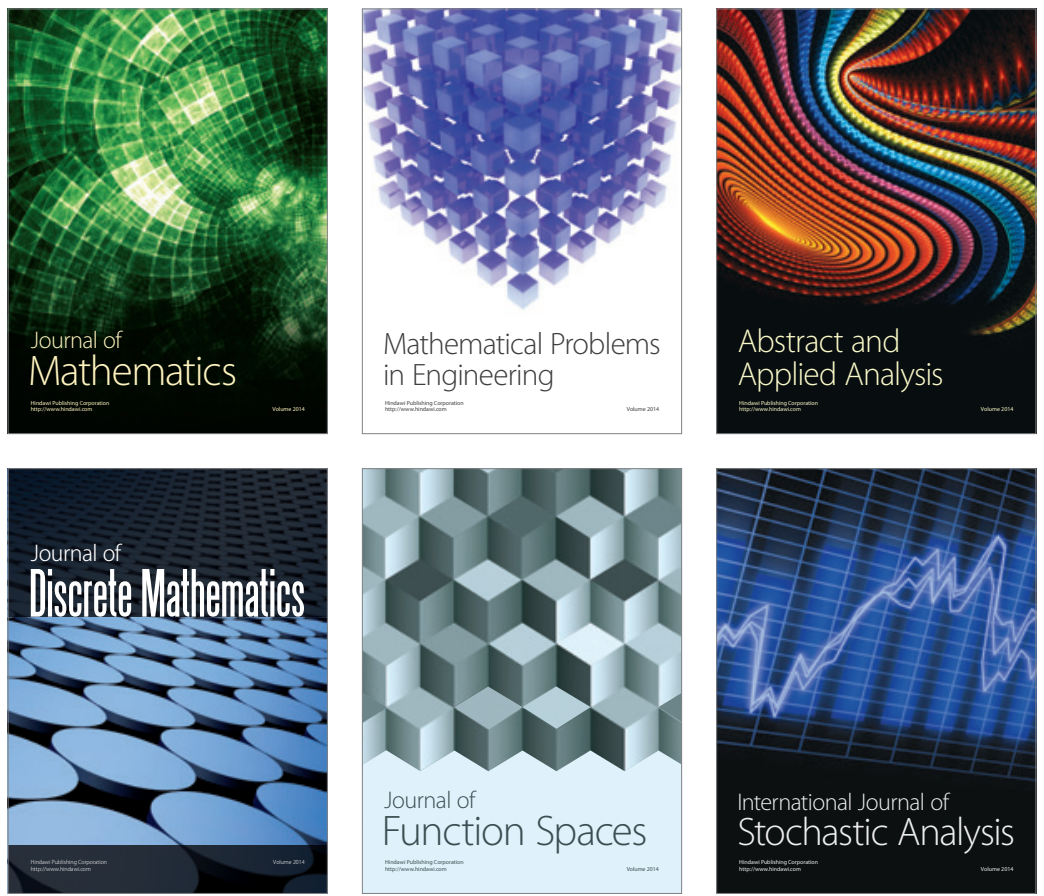

Journal of

Function Spaces

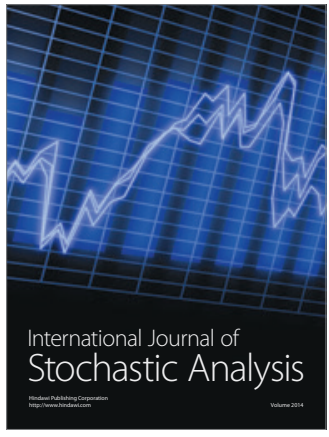

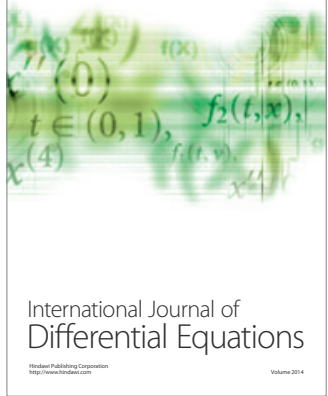
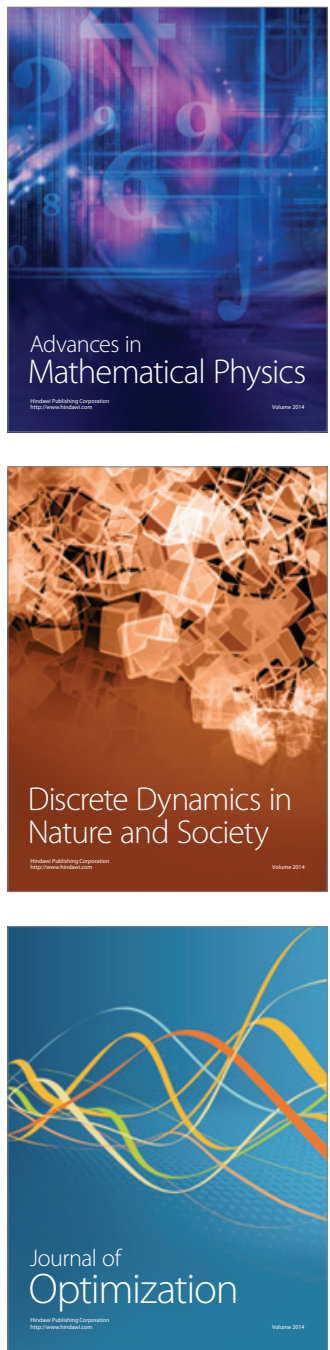